\title{
Cost-effectiveness of the 21-gene recurrence score assay in the context of multifactorial decision making to guide chemotherapy for early-stage breast cancer
}

\author{
Shelby D. Reed, $\mathrm{PhD}^{1,2}$, Michaela A. Dinan, $\mathrm{PhD}^{1}$, Kevin A. Schulman, $\mathrm{MD}^{1,2}$ \\ and Gary H. Lyman, MD2,3
}

Purpose: New evidence is available regarding the utility of the 21-gene recurrence score assay in guiding chemotherapy use for node-negative, estrogen receptor-positive breast cancer. We applied this evidence in a decision-analytic model to re-evaluate the costeffectiveness of the assay.

Methods: We cross-classified patients by clinicopathologic characteristics from the Adjuvant! risk index and by recurrence score risk group. For non-recurrence score-guided treatment, we assumed patients receiving hormonal therapy alone had low-risk characteristics and patients receiving chemotherapy and hormonal therapy had higher-risk characteristics. For recurrence score-guided treatment, we assigned chemotherapy probabilities conditional on recurrence score risk group and clinicopathologic characteristics.

Results: An estimated $40.4 \%$ ofpatientsin the recurrencescore-guided strategy and $47.3 \%$ in the non-recurrence score-guided strategy were

\section{INTRODUCTION}

The Oncotype DX 21-gene recurrence score (RS) assay (Genomic Health; Redwood City, CA) is the most widely used gene signature for guiding the treatment of patients with earlystage, estrogen receptor-positive breast cancer. Using data from two major clinical trials-National Surgical Adjuvant Breast and Bowel Project (NSABP) trials B-14 and B-20,2-the RS assay has been validated as a method for distinguishing between patients with higher and lower risks of distant recurrence, ${ }^{3-5}$ and it has predictive validity in identifying patients who will benefit most from chemotherapy. ${ }^{4,5}$

Previous economic evaluations predicted cost savings with the use of gene expression assays in early-stage breast cancer. ${ }^{6-8}$ These studies relied on normative assumptions about the use of chemotherapy on the basis of the genetic assay results. The researchers assumed that patients at low risk of recurrence (according to assay results) would forgo chemotherapy, whereas all other patients would receive chemotherapy regardless of their other clinical and tumor risk factors. Comparison groups representing "standard practice" in these studies varied. In some studies, all patients were assumed to receive chemotherapy or tamoxifen ${ }^{7}$; in others, the proportions of patients receiving chemotherapy were based on older guidelines that recommended chemotherapy for more than $90 \%$ of patients. ${ }^{6.8}$ expected to receive chemotherapy. The incremental gain in qualityadjusted life-years was 0.16 (95\% confidence interval, $0.08-0.28$ ) with the recurrence score-guided strategy. Lifetime medical costs to the health system were $\$ 2,692(\$ 1,546-\$ 3,821)$ higher with the recurrence score-guided strategy, for an incremental cost-effectiveness ratio of $\$ 16,677 /$ quality-adjusted life-year $(\$ 7,613-\$ 37,219)$. From a societal perspective, the incremental cost-effectiveness was $\$ 10,788$ / quality-adjusted life-year $(\$ 6,840-\$ 30,265)$.

Conclusion: The findings provide supportive evidence for the economic value of the 21 -gene recurrence score assay in node-negative, estrogen receptor-positive breast cancer.

Genet Med 2013:15(3):203-211

Key Words: adjuvant chemotherapy; breast neoplasms; cost-benefit analysis; gene expression; health-care costs; prognosis

Since the publication of these studies, use of gene expression profiling in early-stage breast cancer has expanded to include one-third of eligible patients at some centers. ${ }^{9}$ Nevertheless, concerns persist about the cost-effectiveness of the RS assay, ${ }^{10,11}$ considering that physicians routinely personalize recommendations for chemotherapy according to the patient's pathological and clinical characteristics and independently account for these factors when results of gene expression profiling are available.

Findings from two recent studies provide an opportunity to re-examine the cost-effectiveness of the RS assay. In one study, Lo et al..$^{12}$ reported a prospective, multisite study designed to evaluate treatment recommendations before and after receipt of results from the RS assay. Incorporating the results of this study in a cost-effectiveness model is important to approximate expected costs and outcomes in a real-world setting in which recommendations for chemotherapy depend not only on RS assay results, but also on other clinical and pathological risk factors (e.g., pre-assay recommendations). In the other study, Tang et al. ${ }^{5}$ analyzed patient-level data from NSABP trials B-14 and B-20 to compare the prognostic and predictive validity of the RS assay and Adjuvant!, a decision aid that incorporates information on patients' clinical and tumor characteristics, such as age, tumor size, node involvement, and hormone and human epidermal growth factor receptor (HER-2) status, with

${ }^{1}$ Duke Clinical Research Institute, Duke University School of Medicine, Durham, North Carolina, USA; ${ }^{2}$ Duke Cancer Institute, Duke University School of Medicine, Durham, North Carolina, USA; ${ }^{3}$ Department of Medicine, Duke University School of Medicine, Durham, North Carolina, USA. Correspondence: Shelby D. Reed (shelby.reed@duke.edu) 
regard to distant recurrence. Both the RS assay and Adjuvant! were strong prognostic indicators of distant recurrence; however, only the RS assay was a significant predictor of benefit from chemotherapy.

Although many physicians do not use Adjuvant! to guide treatment recommendations, the tool incorporates many of the same clinicopathologic factors that are most influential in treatment recommendation $s^{13}$ and broadly agrees with recommendations from multidisciplinary teams. ${ }^{14}$ Therefore, we sought to incorporate new evidence from Lo et al. ${ }^{12}$ and Tang et al. ${ }^{5}$ to re-evaluate the cost-effectiveness of a strategy in which RS assay results are available along with other clinicopathologic characteristics (i.e., the RS-guided strategy) as compared with a strategy limited to clinicopathologic characteristics (i.e., the non-RS-guided strategy) to guide the use of chemotherapy for node-negative, estrogen receptor-positive breast cancer in the United States.

\section{Model structure}

\section{MATERIALS AND METHODS}

We developed a decision-analytic model to estimate costs, survival, and quality-adjusted survival for RS-guided and
non-RS-guided strategies. The model categorized patients according to the clinicopathologic characteristics in the Adjuvant! risk index using cut points reported by Tang et al. ${ }^{5}$ (i.e., low risk, $\leq 5.5$; intermediate risk, $>5.5$ and $\leq 11.9$; and high risk, >11.9; Figure 1). Using conditional probabilities, we further stratified patients according to previously defined RS risk groups to allow for a fair comparison by ensuring that underlying risk profiles with both treatment strategies were the same. ${ }^{3,4}$ The impact of the RS-guided strategy was to selectively guide the use of chemotherapy beyond the risk information conveyed by clinicopathologic characteristics. Therefore, only the probabilities corresponding to chemotherapy and hormonal therapy differed between the strategies (Table 1).

In the base-case analysis for the non-RS-guided strategy, we assumed that patients categorized as being at intermediate or high risk according to clinicopathologic characteristics (i.e., Adjuvant!) would receive chemotherapy followed by hormonal therapy and patients with low-risk clinicopathologic characteristics would receive hormonal therapy alone. For the RS-guided strategy, we incorporated evidence from Lo et al. ${ }^{12}$ indicating that a physician's recommendation for chemotherapy depends

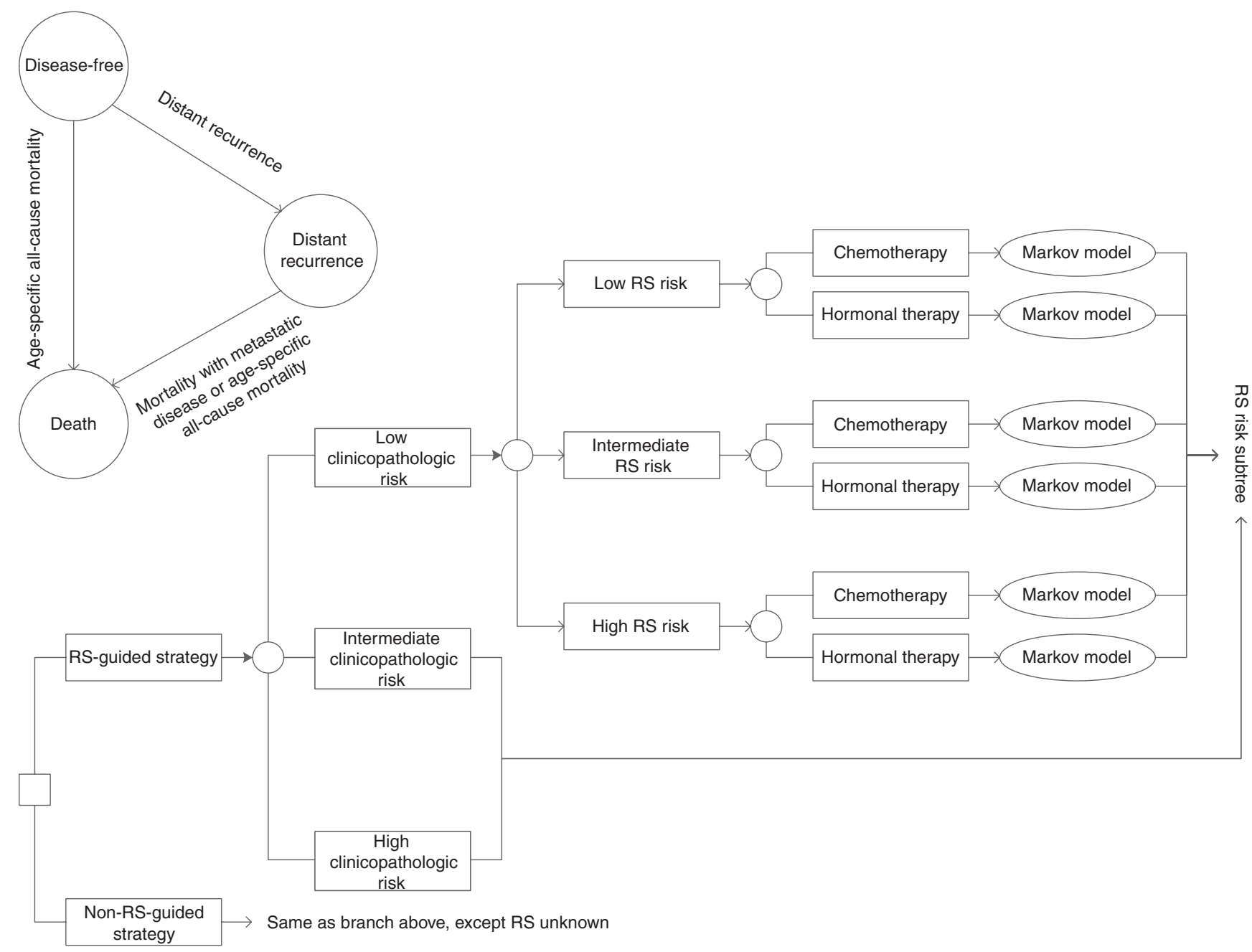

Figure 1 Schematic of the decision tree and Markov model. RS, recurrence score. 
Table 1 Model parameters in the base-case analysis

\section{Parameter}

Proportion of patients by clinicopathologic risk group ${ }^{a}$

Low

Intermediate

High

Proportion of patients by RS risk group and clinicopathologic risk group ${ }^{\text {a }}$

Low clinicopathologic risk

RS low risk

RS intermediate risk

RS high risk

Intermediate clinicopathologic risk ${ }^{a}$

RS low risk

RS intermediate risk

RS high risk

High clinicopathologic risk ${ }^{\mathrm{a}}$

RS low risk

RS intermediate risk

RS high risk

Proportion of patients receiving chemotherapy by clinicopathologic risk group ${ }^{a}$

Low clinicopathologic risk

Intermediate clinicopathologic risk

High clinicopathologic risk

Proportion of patients receiving chemotherapy by RS risk group and

clinicopathologic risk group ${ }^{\mathrm{a}, \mathrm{b}}$

Low clinicopathologic risk ${ }^{c}$

$$
\begin{aligned}
& \text { RS low risk } \\
& \text { RS intermediate risk } \\
& \text { RS high risk }
\end{aligned}
$$

Intermediate or high clinicopathologic risk ${ }^{\mathrm{d}}$
RS low risk
RS intermediate risk

RS high risk

10-year distant recurrence-free with hormone therapy

RS low risk

RS intermediate risk

RS high risk

Relative risk with chemotherapy on distant recurrence

RS low risk

RS intermediate risk

RS high risk

5-year mortality after distant recurrence

Discount rate for costs and QALYS

Health state utilities
Mean (SE)

$0.527(0.020)$

$0.186(0.007)$

$0.287(0.011)$

$0.614(0.033)$

$0.239(0.013)$

$0.148(0.008)$

$0.460(0.041)$

$0.194(0.017)$

$0.347(0.031)$

$0.339(0.024)$

$0.214(0.015)$

$0.448(0.032)$

0

1.0

1.0

(n)
Tang et al. ${ }^{5}$

Tang et al. ${ }^{5}$

Source

Tang et al. ${ }^{5}$

Tang et al. ${ }^{5}$

Assumption

Assumption

Lo et al. ${ }^{12}$

$0.045(0.044)$

$0.095(0.064)$

$1.0(0)$

Lo et $a^{12}$

$0.250(0.108)$

$0.619(0.106)$

$1.0(0)$

$0.968(0.016)$

$0.909(0.043)$

$0.605(0.073)$

Paik et al. ${ }^{4}$

$1.31\left(0.57^{\mathrm{e}}\right)$

$0.61\left(0.56^{\mathrm{e}}\right)$

$0.26\left(0.31^{\mathrm{e}}\right)$

$0.766(0.01)$

$3 \%$ per year

Paik et al. ${ }^{4}$

Chemotherapy in the first year

$0.48(0.06)$

$0.68(0.06)$

Hormonal therapy

QALY, quality-adjusted life-year; RS, 21-gene recurrence score assay.

${ }^{a}$ Clinicopathologic risk groups based on Adjuvant! risk index scores with cut off points defined by Tang et al. ${ }^{5}$ (i.e., $\leq 5.5$, low risk; intermediate risk, $>5.5$ and $\leq 11.9$; high risk, >11.9). " Assumes that the initial recommendation in Lo et al..$^{12}$ reported as "equipoise" represents chemotherapy. We performed sensitivity analyses to evaluate the impact when "equipoise" was assumed to represent hormone therapy. 'Assumes that the initial recommendation in Lo et al. ${ }^{12}$ reported as hormonal therapy corresponds to patients who would be classified as being at low clinicopathologic risk. We performed sensitivity analyses to evaluate the assumption that the initial recommendation of hormonal therapy represented patients in the low and intermediate clinicopathologic risk groups. We also performed sensitivity analyses with the assumption that treatment recommendations based on RS risk group were not conditional on the initial recommendation based on clinicopathologic risk groups (probability of chemotherapy assuming "equipoise" in Lo et al. ${ }^{12}$ represents chemotherapy RS low risk at 13.2\%, RS intermediate risk at 35.7\%, and RS high risk at $100 \%$ ).

${ }^{\mathrm{d}}$ Assumes that the initial recommendation in Lo et al. ${ }^{12}$ reported as chemotherapy or "equipoise" corresponds to patients who would be classified as intermediate or high clinicopathologic risk. eRepresents the standard error of log relative risk. fUpdated to 2011 values using the consumer price index for medical care. ${ }^{40} \mathrm{gRepresents}$ the 10 -year cost for estrogen receptor-positive patients with distant recurrence discounted at 3\%.

Table 1 Continued on next page 
Table 1 Continued.

\begin{tabular}{|c|c|c|}
\hline Parameter & Mean (SE) & Source \\
\hline Remission & $0.68(0.06)$ & \\
\hline Distant recurrence & $0.42(0.06)$ & \\
\hline 21-Gene recurrence score assay & 4,075 & $\begin{array}{l}\text { Genomic Health } 2010 \text { Annual } \\
\text { Report }\end{array}$ \\
\hline Chemotherapy, first year & $16,947^{\mathrm{a}}(1,655)$ & Oestreicher et al. ${ }^{17}$ \\
\hline Monitoring and follow-up during remission, annually for up to 10 years & $1,108^{f}(61)$ & Hensley et al. ${ }^{18}$ \\
\hline Distant recurrence, one-time cost ${ }^{\dagger}$ & $17,478^{f}(2,444)$ & Stokes et al. ${ }^{19}$ \\
\hline \multicolumn{3}{|l|}{ Indirect costs, \$ } \\
\hline Absence from work attributable to chemotherapy & 12,686 & $\begin{array}{l}\text { Drolet et al. }{ }^{20} \text {; Bureau of Labor } \\
\text { Statistics, National Compensation } \\
\text { Survey }\end{array}$ \\
\hline
\end{tabular}

QALY, quality-adjusted life-year; RS, 21-gene recurrence score assay.

a Clinicopathologic risk groups based on Adjuvant! risk index scores with cut off points defined by Tang et al. ${ }^{\text {5i.e., }} \leq 5.5$, low risk; intermediate risk, $>5.5$ and $\leq 11.9$; high risk, >11.9). "'Assumes that the initial recommendation in Lo et al. ${ }^{12}$ reported as "equipoise" represents chemotherapy. We performed sensitivity analyses to evaluate the impact when "equipoise" was assumed to represent hormone therapy. 'Assumes that the initial recommendation in Lo et al. ${ }^{12}$ reported as hormonal therapy corresponds to patients who would be classified as being at low clinicopathologic risk. We performed sensitivity analyses to evaluate the assumption that the initial recommendation of hormonal therapy represented patients in the low and intermediate clinicopathologic risk groups. We also performed sensitivity analyses with the assumption that treatment recommendations based on RS risk group were not conditional on the initial recommendation based on clinicopathologic risk groups (probability of chemotherapy assuming "equipoise" in Lo et al. ${ }^{12}$ represents chemotherapy RS low risk at 13.2\%, RS intermediate risk at 35.7\%, and RS high risk at $100 \%$ ).

${ }^{\mathrm{d}}$ Assumes that the initial recommendation in Lo et al. ${ }^{12}$ reported as chemotherapy or "equipoise" corresponds to patients who would be classified as intermediate or high clinicopathologic risk. ${ }^{e}$ Represents the standard error of log relative risk. ${ }^{f}$ Updated to 2011 values using the consumer price index for medical care. ${ }^{40}{ }^{9}$ Represents the 10 -year cost for estrogen receptor-positive patients with distant recurrence discounted at 3\%.

on both the patient's RS risk group and whether the physician had recommended chemotherapy based on clinicopathologic characteristics before receiving RS assay results. The treatment effect of adjuvant chemotherapy on distant recurrence was conditional on the RS risk classification. ${ }^{4}$

After stratification by risk and treatment, hypothetical patients cycled through a Markov model representing the incidence of distant recurrence, death from breast cancer, and death from other causes (Figure 1). ${ }^{3}$ The cycle length was 6 months. Progression from distant recurrence to death was based on data from a Surveillance, Epidemiology, and End Results (SEER) cohort. ${ }^{15}$ We modeled the probability of death not attributable to breast cancer using age-specific annual mortality rates for women in the United States. ${ }^{16}$ In the base-case analysis, we assumed that patients were aged 55 years at the time of diagnosis. ${ }^{12}$

\section{Costs and utility weights}

We performed the analysis both from the US health-system perspective inclusive of all direct medical costs and from the societal perspective inclusive of all direct medical costs plus patients' time costs.

We assigned costs attributable to chemotherapy to the first cycle in the Markov model, and we assigned biannual costs of hormonal therapy beginning in the third 6-month cycle (Table 1). ${ }^{17}$ For hormonal therapy, we assigned costs for tamoxifen across 5 years. We assigned medical costs associated with monitoring and follow-up for up to 10 years or until the diagnosis of distant recurrence. ${ }^{18}$ On development of distant recurrence, we assigned attributable costs estimated from SEER-Medicare data. ${ }^{19}$

We calculated patient time costs associated with chemotherapy from a study of the cumulative time lost from work over 3 years among women who received adjuvant chemotherapy $(9.5$ months) as compared with women who did not (5.4 months). ${ }^{20}$ Time associated with distant recurrence was based on the time that patients with breast cancer spent in their last year of life receiving medical care. ${ }^{21}$ We valued patient time on the basis of wage rates for US civilian workers. ${ }^{22}$

To account for differential health-related quality of life, we assigned utility weights reported by Schleinitz et al., ${ }^{23}$ representing different stages of and treatments for breast cancer.

\section{Sensitivity analyses}

To perform a probabilistic sensitivity analysis, we assigned distributions to model parameters to represent the uncertainty associated with the point estimates. As recommended for modeling second-order uncertainty, we used Dirichlet distributions to model multinomial parameters, beta distributions to model probabilities and utility weights, and normal distributions to model costs and log-transformed relative risks. ${ }^{24}$ We applied a Monte Carlo simulation to generate 1,000 runs and identified the 25 th and 975 th ranks as the corresponding $95 \%$ confidence intervals (CIs).

We also performed one-way sensitivity analyses. We varied the age at diagnosis and evaluated the impact of changes to assumptions regarding treatment decisions and the target population. We also extended the time period during which 
patients were at risk for recurrence, varied the discount rate, applied utility weights from other sources, ${ }^{25,26}$ applied wages of government workers to value patient time, ${ }^{22}$ doubled the cost assigned for chemotherapy, assigned costs for aromatase inhibitors instead of tamoxifen, and applied higher costs for distant recurrence. ${ }^{19,27}$

\section{RESULTS}

For an estimated $27.9 \%$ of patients, treatment recommendations changed after the incorporation of RS information. An estimated $40.4 \%$ of patients in the RS-guided strategy and $47.3 \%$ in the non-RS-guided strategy were expected to receive adjuvant chemotherapy, a $15 \%$ relative reduction (Table 2). During the first year, total direct medical costs in the RS-guided strategy were an estimated $\$ 11,632$, as compared with $\$ 8,735$ in the non-RS-guided strategy—a $\$ 2,897$ increase.

Estimated rates of recurrence at 10 years were $6.8 \%$ with the RS-guided strategy and $8.9 \%$ with the non-RS guided strategy. Targeted use of chemotherapy in the RS-guided strategy was associated with expected gains of 0.19 life-years and 0.16 quality-adjusted life-years (QALYs) (Table 3). From a healthsystem perspective, lifetime direct medical costs were an estimated $\$ 2,692$ higher with the RS-guided strategy, resulting in incremental cost-effectiveness ratios of $\$ 14,059$ per life-year saved (95\% CI, \$6,840-\$28,912) and \$16,677 per QALY (95\% CI, $\$ 7,613-\$ 37,219)$. From a societal perspective that incorporated lower patient time costs of $\$ 950$ per patient, the incremental cost-effectiveness ratios were $\$ 9,095$ per life-year saved (95\% CI, dominant \$23,397) and \$10,788 per QALY (95\% CI, $\$ 6,840-\$ 30,265)$. Probabilistic sensitivity analysis indicated that more than $99 \%$ of the incremental cost-effectiveness ratios generated in Monte Carlo simulations were less than $\$ 50,000$ per life-year saved and per QALY (Supplementary Figure S1 online), consistent with the corresponding 95\% CIs.

In sensitivity analyses, the results were relatively unaffected by changes in individual model assumptions and inputs

Table 2 Treatment probabilities cross-classified by RS-guided and non-RS-guided strategies

\begin{tabular}{lccc|}
\hline $\begin{array}{l}\text { Non-RS-guided } \\
\text { strategy }\end{array}$ & $\begin{array}{c}\text { Hormonal } \\
\text { therapy }\end{array}$ & $\begin{array}{c}\text { Chemo- } \\
\text { therapy }\end{array}$ & Total \\
\hline Hormonal therapy, \% & 42.2 & 10.5 & 52.7 \\
\hline Chemotherapy, \% & 17.4 & 29.9 & 47.3 \\
\hline Total, \% & 59.6 & 40.4 & 100.0 \\
\hline
\end{tabular}

$\mathrm{RS}$, recurrence score.
(Table 4). Changes to assumptions necessary to apply the findings from Lo et al. ${ }^{9}$ on the use of chemotherapy had little impact. However, when we limited the target population to patients with intermediate- or high-risk clinical characteristics, $100 \%$ of patients in the non-RS-guided strategy were assumed to receive chemotherapy. In this scenario, there was a $37 \%$ absolute reduction in the use of chemotherapy, resulting in lower direct medical costs with the RS-guided strategy as compared with the non-RS-guided strategy ( $\$ 24,857$ vs. $\$ 27,121)$. When we assumed that all patients across clinicopathologic risk groups in the non-RS-guided strategy would receive chemotherapy, expected savings with the RS-guided strategy were $\$ 5,945$ per patient from the health-system perspective and $\$ 7,526$ from the societal perspective.

As expected, assigning higher costs to chemotherapy and distant recurrence improved the cost-effectiveness of the RS-guided strategy. To reach cost neutrality, costs associated with distant recurrence would have to approximate $\$ 165,000$ per case from the health-system perspective or $\$ 113,000$ from the societal perspective.

\section{DISCUSSION}

Our findings provide evidence regarding the economic value of the 21-gene RS assay in the setting of estrogen receptor-positive, node-negative breast cancer. In the base-case analysis, an estimated $47.3 \%$ of patients in the non-RS-guided strategy and $40.4 \%$ in the RS-guided strategy would receive adjuvant chemotherapy. This $15 \%$ reduction generated $\sim 1,200$ in savings in direct medical costs, which offset approximately one-quarter of the cost of the RS assay $(\$ 4,075)$, a net increase of $\sim \$ 2,900$. From the patient perspective, indirect costs were $\sim \$ 950$ lower with the RS-guided strategy. When we combined estimated cost increases with expected gains in quality-adjusted survival with the RS-guided strategy, the incremental cost-effectiveness was $\sim 17,000$ per QALY from the health-system perspective and $\$ 11,000$ per QALY from the societal perspective.

When we modeled the non-RS-guided strategy in the basecase analysis, we assumed that none of the patients with lowrisk clinicopathologic characteristics would receive chemotherapy, consistent with recommendations for patients with Adjuvant! risk index $\leq 5.5$. This assumption favored the nonRS-guided strategy on two accounts. First, the availability of RS information in the RS-guided strategy could only increase (from zero) the proportion of patients receiving chemotherapy. Second, among the low RS risk group, the receipt of chemotherapy led to lower quality-adjusted survival because

Table 3 Results of the base-case analysis

\begin{tabular}{lccc} 
Variable & RS-guided strategy & Non-RS-guided strategy & Difference \\
\hline Life-years, discounted $(95 \% \mathrm{Cl})$ & $15.02(14.66-15.24)$ & $14.82(14.46-15.07)$ & $0.19(0.09-0.32)$ \\
Quality-adjusted life-years, discounted, $(95 \% \mathrm{Cl})$ & $10.09(8.240-11.79)$ & $9.93(8.12-11.60)$ & $0.16(0.08-0.28)$ \\
Direct costs, discounted $(95 \% \mathrm{Cl}), \$$ & $21,090(19,306-23,139)$ & $18,398(16,535-20,448)$ & $2,692(1,546-3,821)$ \\
Indirect costs, discounted $(95 \% \mathrm{CI}), \$$ & $5,307(4,615-6,178)$ & $6,257(5,794-6,745)$ & $-950(-1,732$ to -111$)$ \\
Total costs, discounted $(95 \% \mathrm{Cl}), \$$ & $26,397(24,073-28,957)$ & $24,656(22,599-26,887)$ & $1,741(-85$ to 3,710$)$ \\
\hline
\end{tabular}

$\mathrm{Cl}$, confidence interval; $\mathrm{RS}$, recurrence score. 
Table 4 Results of sensitivity analyses

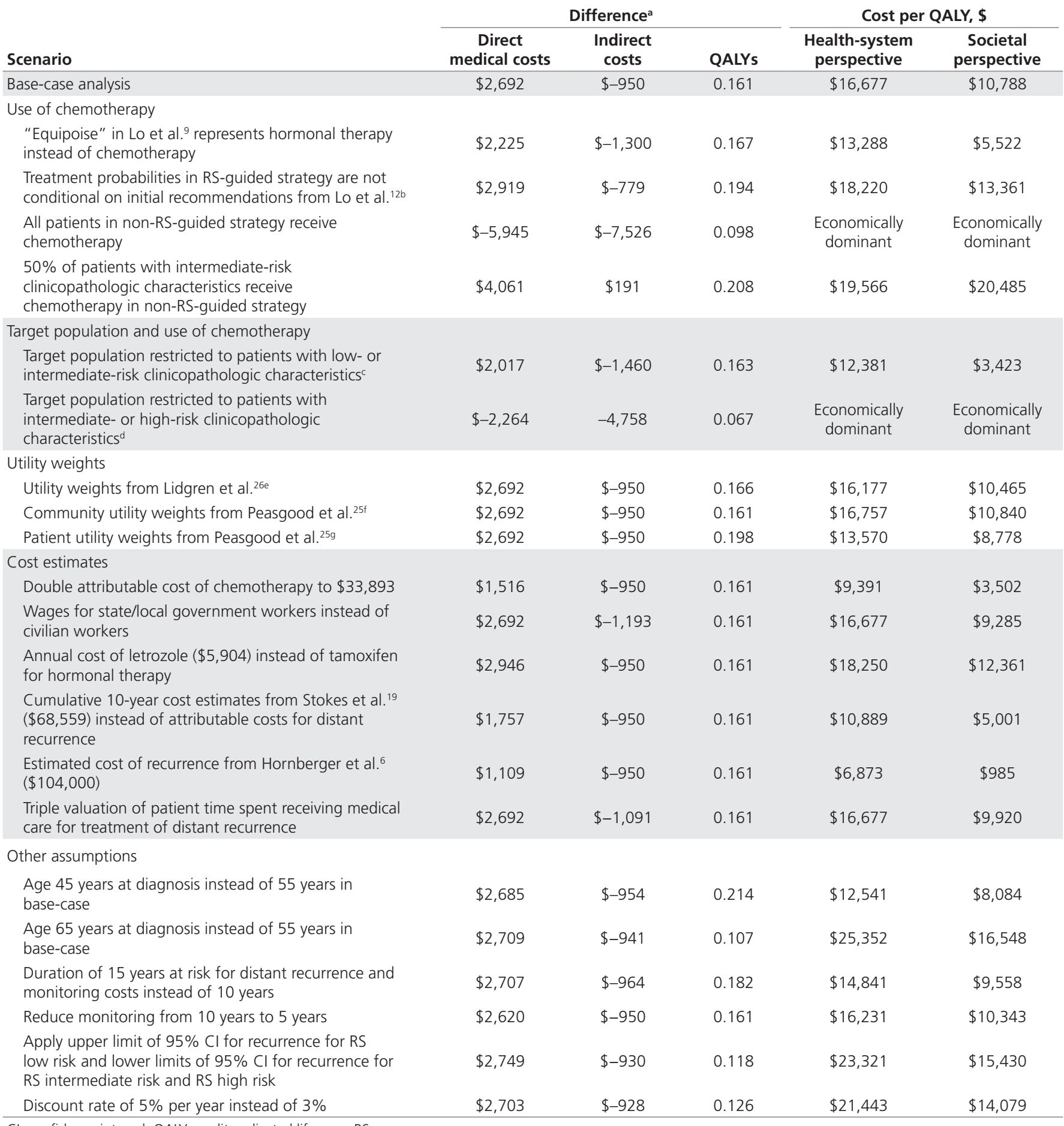

$\mathrm{Cl}$, confidence interval; QALY, quality-adjusted life-year; RS, recurrence score.

aRS-guided strategy minus non-RS-guided strategy. bProbabilities of chemotherapy assuming that "equipoise" in Lo et al. ${ }^{9}$ represents chemotherapy: low RS, $13.2 \%$; intermediate RS, 35.7\%; high RS, 100\%. 'Assumes a 50:50 ratio of patients with low- and intermediate-risk clinicopathologic characteristics (i.e., 50\% of patients in the non-RS-guided strategy would receive chemotherapy and 39.0\% of patients in the RS-guided strategy would receive chemotherapy). ${ }^{\mathrm{d} A s s u m e s}$ a $50: 50$ ratio of patients with intermediate- and high-risk clinicopathologic characteristics (i.e., $100 \%$ of patients in the non-RS-guided strategy would receive chemotherapy and $62.3 \%$ of patients in the RS-guided strategy would receive chemotherapy). ${ }^{~}$ Chemotherapy, 0.62 ; hormonal therapy, 0.744 ; remission, 0.779 ; recurrence, 0.685 . ${ }^{\top}$ The utility weight for chemotherapy (0.637) was based on regression model 2 published by Peasgood et al. ${ }^{25}$ for early-stage breast cancer, which corresponded to chemotherapy with toxicity or nausea and vomiting as elicited from a standard gamble. The utility weights for hormonal therapy and remission (both 0.701$)$ corresponded to chemotherapy over 1 year, as elicited from a standard gamble. The utility weight for distant recurrence $(0.373)$ was based on regression model 2 for metastatic breast cancer with unspecified treatment response, as elicited from a standard gamble. ${ }^{9}$ Chemotherapy, 0.846 ; hormonal therapy, 0.91; remission, 0.91 ; distant recurrence, 0.616. 
of the greater hazard of distant recurrence reported for this risk group. ${ }^{4}$ An equally important assumption for the nonRS-guided strategy was that all patients with intermediate- or high-risk clinicopathologic characteristics (i.e., Adjuvant! risk index $>5.5$ ) would receive chemotherapy. Therefore, the addition of RS information could only lead to a reduction in the use of chemotherapy for these patients, improving the costeffectiveness of the RS-guided strategy through lower chemotherapy costs.

The extent to which these two countervailing effects changed the overall number of patients receiving chemotherapy was a function of the distribution of patients across risk groups. Therefore, an important consideration is whether patients studied by Tang et al. ${ }^{5}$ are representative of patients who receive the RS assay in practice. The proportions of patients with a low RS in NSABP B-14 (51\%) and in observational studies are similar (Supplementary Table S1 online). ${ }^{12,27-32}$ However, approximately one-quarter of patients in NSABP B-14 had a high-risk $\mathrm{RS}$, a larger representation as compared with patients receiving the RS assay in practice (Supplementary Table S1 online). ${ }^{12,27-32}$ This finding may be attributable to physicians having less uncertainty about the use of chemotherapy when a patient presents with several high-risk characteristics. Nevertheless, our sensitivity analyses revealed that reducing the proportion of patients in the high-risk RS group had relatively little impact on estimates of cost-effectiveness.

The study by Lo et al. ${ }^{12}$ allowed us to model treatment recommendations in the setting of knowledge about clinicopathologic risk characteristics alone and with the addition of genetic risk information. Therefore, we believe the analysis is representative of real-world decision making. Observational studies have shown that treatment recommendations based on clinical judgment (i.e., before RS information) influence treatment recommendations after RS information is provided. ${ }^{12,28,30}$ For example, among patients with intermediate RS, chemotherapy was recommended for $10 \%$ when the initial recommendation was for hormonal therapy, as compared with $62 \%$ when the initial recommendation was for chemotherapy. ${ }^{12}$

Although our assumption that patients with low-risk clinicopathologic characteristics forgo chemotherapy while patients at higher risk receive chemotherapy in the non-RS-guided strategy is open to argument, our overall estimate of chemotherapy in $47.3 \%$ of patients is similar to rates reported for patients without RS information (e.g., $48.3 \%,{ }^{12} 46.7 \%,{ }^{28}$ and $48.5 \%{ }^{30}$ ). Nevertheless, it is important to acknowledge the influence of assumptions about the use of chemotherapy in the non-RSguided strategy. When we assumed universal chemotherapy with the non-RS-guided strategy, use of the RS assay led to expected savings of $\$ 6,000$ per patient with gains in quality-adjusted survival. When we assumed that only $50 \%$ of patients in the lowrisk group would receive chemotherapy with the non-RS-guided strategy, the RS assay led to cost savings of more than $\$ 2,200$ per patient with gains in quality-adjusted survival. The ideal data source would have provided treatment recommendations stratified on the basis of Adjuvant! before and after the availability of RS information. However, we identified only one such study of 29 patients. ${ }^{33}$ Other studies that examined cross-classification by Adjuvant! and RS did not provide information on treatment recommendations before the receipt of RS information..$^{31,32}$

Early economic evaluations of the RS assay reported cost savings and gains in QALYs. ${ }^{6-8}$ These studies projected greater cost savings with the RS assay than did our study because of assumptions that the use of chemotherapy without RS risk information (i.e., standard care) ranged from 92 to $100 \%$ and that the use of chemotherapy was entirely governed according to RS risk categories with $0 \%$ use in RS low-risk groups and $100 \%$ use in RS intermediate- and RS high-risk groups. ${ }^{6,7}$ These studies also did not model the differential treatment effects of chemotherapy across RS risk groups that were later reported by Paik et al. ${ }^{4}$

More recent studies portraying real-world decision making had more variable results. ${ }^{27,29,34}$ Our findings are consistent with a study in Israel ( $\$ 10,770$ per QALY $)^{29}$ and a study in Japan $(\$ 3,848 \text { per QALY })^{35}$ but more optimistic than a study in Canada ( $\$ 63,000$ per QALY in 2008 Canadian dollars), ${ }^{34}$ although differences in costs and practice patterns limit the validity of crosscountry comparisons. ${ }^{36}$ Our findings are less optimistic than those from a study in a US managed-care population in which cost savings and QALY gains were reported. ${ }^{27}$ In that analysis, $50-60 \%$ of patients were expected to receive chemotherapy without RS information. With a $27 \%$ reduction in chemotherapy with the RS assay, chemotherapy was expected in about 37 , 40 , and $44 \%$ in the low-, intermediate, and high-risk RS groups, respectively. In our model for the RS-guided strategy, chemotherapy was used in 12,32 , and $100 \%$ of the respective RS risk groups-rates that appear to be consistent with observational studies (Supplementary Table S1 online). ${ }^{30-32,37}$

Another methodological difference is the cost of distant recurrence. Whereas the managed-care analysis assigned a cost of $\$ 104,000$ per case,,$^{27}$ we applied an estimate of $\sim \$ 17,500$, the difference in 10-year discounted costs between patients who experienced distant recurrence as compared with patients who did not. ${ }^{19}$ Although this cost estimate may appear to be low, the estimate is the net of background medical costs and was developed specifically for application in cost-effectiveness analyses. ${ }^{38}$ The estimate is not ideal because it relied on 1991-2002 SEERMedicare data and may not reflect current treatment patterns. However, our threshold analyses showed that costs associated with distant recurrence would have to surpass $\$ 165,000$ to offset the cost of the RS assay.

Other limitations may also influence the real-world costeffectiveness of the RS assay. First, patients often do not follow their physicians' treatment recommendations. ${ }^{12}$ Also, much of the variability in the results across probabilistic sensitivity analyses stemmed from the wide confidence intervals representing the impact of chemotherapy on distant recurrence in the low RS and intermediate RS groups. ${ }^{4}$ Results from the Trial Assigning Individualized Options for Treatment (TAILORx) will provide more precise estimates of treatment effect for patients with intermediate RS. In addition, the model does not allow for direct variation of measures of the assay's diagnostic 
accuracy, such as positive predictive value. However, the model allows for representations of both accurate and inaccurate predictions of recurrence.

In conclusion, we estimate that use of the RS assay will reduce the use of chemotherapy from 47.3 to $40.4 \%$. Although this reduction is conservative, targeted use of chemotherapy with the RS assay is associated with cost-effectiveness ratios of $\sim \$ 17,000$ per QALY from the health-system perspective and $\$ 11,000$ per QALY from the societal perspective, both well below commonly cited thresholds of $\$ 50,000-\$ 100,000$ per QALY used for gauging the cost-effectiveness of health technologies.

\section{SUPPLEMENTARY MATERIAL}

Supplementary material is linked to the online version of the paper at http://www.nature.com/gim

\section{ACKNOWLEDGMENTS}

This work was supported by a research agreement between Genomic Health, Inc, and Duke University. G.H.L. was also supported by grant 5UC2CA148041-02 from the National Cancer Institute.

Damon M. Seils, MA, Duke University, provided editorial assistance and prepared the manuscript. Mr Seils did not receive compensation for his assistance apart from his employment at the institution where the work was conducted.

\section{DISCLOSURE}

G.H.L. reported receiving previous research support from Genomic Health. No other disclosures were reported. S.D.R. and K.A.S. have made available online detailed listings of financial disclosures (http://www.dcri.duke.edu/about-us/conflict-of-interest/).

\section{REFERENCES}

1. Fisher B, Costantino J, Redmond C, et al. A randomized clinical trial evaluating tamoxifen in the treatment of patients with node-negative breast cancer who have estrogen-receptor-positive tumors. N Engl J Med 1989;320:479-484.

2. Fisher B, Dignam J, Wolmark N, et al. Tamoxifen and chemotherapy for lymph node-negative, estrogen receptor-positive breast cancer. J Natl Cancer Inst 1997;89:1673-1682

3. Paik S, Shak S, Tang G, et al. A multigene assay to predict recurrence of tamoxifen-treated, node-negative breast cancer. N Engl J Med 2004;351:2817-2826

4. Paik S, Tang G, Shak S, et al. Gene expression and benefit of chemotherapy in women with node-negative, estrogen receptor-positive breast cancer. J Clin Oncol 2006;24:3726-3734.

5. Tang G, Shak S, Paik S, et al. Comparison of the prognostic and predictive utilities of the 21-gene Recurrence Score assay and Adjuvant! for women with node-negative, ER-positive breast cancer: results from NSABP B-14 and NSABP B-20. Breast Cancer Res Treat 2011;127:133-142.

6. Hornberger J, Cosler LE, Lyman GH. Economic analysis of targeting chemotherapy using a 21-gene RT-PCR assay in lymph-node-negative, estrogen-receptor-positive, early-stage breast cancer. Am J Manag Care 2005;11:313-324.

7. Lyman GH, Cosler LE, Kuderer NM, Hornberger J. Impact of a 21-gene RT-PCR assay on treatment decisions in early-stage breast cancer: an economic analysis based on prognostic and predictive validation studies. Cancer 2007;109:1011-1018.

8. Oestreicher N, Ramsey SD, Linden HM, et al. Gene expression profiling and breast cancer care: what are the potential benefits and policy implications? Genet Med 2005;7:380-389.
9. Hassett MJ, Silver SM, Hughes ME, et al. Adoption of gene expression profile testing and association with use of chemotherapy among women with breast cancer. J Clin Oncol 2012:30:2218-2226.

10. Evaluation of Genomic Applications in Practice and Prevention (EGAPP) Working Group. Recommendations from the EGAPP Working Group: can tumor gene expression profiling improve outcomes in patients with breast cancer? Genet Med 2009;11:66-XX.

11. Marchionni L, Wilson RF, Marinopoulos SS, et al. Impact of gene expression profiling tests on breast cancer outcomes. Evid Rep Technol Assess (Full Rep) 2007;160:1-105.

12. Lo SS, Mumby PB, Norton J, et al. Prospective multicenter study of the impact of the 21 -gene recurrence score assay on medical oncologist and patient adjuvant breast cancer treatment selection. J Clin Onco/ 2010;28:1671-1676.

13. Elder EE, Hay SB, Moore K. Factors influencing treatment recommendations in node-negative breast cancer. J Oncol Pract 2011;7:26-30.

14. Nowak V, Ah-See M, Ravichandran D. Breast cancer multidisciplinary team adjuvant therapy decision making and adjuvant! Online. Clin Oncol (R Coll Radiol) 2010;22:87-88.

15. National Cancer Institute. SEER Cancer Statistics Review 1975-2008 Figure 4.11. http://seer.cancer.gov/csr/1975_2008/index.html. Accessed 4 August 2011

16. Arias E. United States life tables, 2004. National Vital Statistics Report 56:140. http://www.cdc.gov/nchs/data/nvsr/nvsr56/nvsr56_09.pdf. Accessed December 2011.

17. Oestreicher N, Ramsey SD, McCune JS, Linden HM, Veenstra DL. The cost of adjuvant chemotherapy in patients with early-stage breast carcinoma. Cancer 2005;104:2054-2062.

18. Hensley ML, Dowell J, Herndon JE II, et al. Economic outcomes of breast cancer survivorship: CALGB study 79804. Breast Cancer Res Treat 2005;91:153-161.

19. Stokes ME, Thompson D, Montoya EL, Weinstein MC, Winer EP, Earle CC. Ten-year survival and cost following breast cancer recurrence: estimates from SEER-medicare data. Value Health 2008;11:213-220.

20. Drolet M, Maunsell E, Mondor M, et al. Work absence after breast cancer diagnosis: a population-based study. CMAJ 2005;173:765-771.

21. Yabroff KR, Davis WW, Lamont EB, et al. Patient time costs associated with cancer care. J Natl Cancer Inst 2007;99:14-23.

22. US Bureau of Labor Statistics. National Compensation Survey: Table 1Summary: Mean hours earnings and weekly hours for selected worker and establishment characteristics. http://www.bls.gov/ncs/ocs/sp/nctb1344.pdf. Accessed 12 January 2012

23. Schleinitz MD, DePalo D, Blume J, Stein M. Can differences in breast cancer utilities explain disparities in breast cancer care? J Gen Intern Med 2006;21:1253-1260

24. Briggs A, Sculpher M, Claxton K. Decision Modeling for Health Economic Evaluation. Oxford University Press: Oxford, UK, 2006.

25. Peasgood T, Ward SE, Brazier J. Health-state utility values in breast cancer. Expert Rev Pharmacoecon Outcomes Res 2010;10:553-566.

26. Lidgren M, Wilking N, Jönsson B, Rehnberg C. Health related quality of life in different states of breast cancer. Qual Life Res 2007;16:1073-1081.

27. Hornberger J, Chien R, Krebs K, Hochheiser L. US insurance program's experience with a multigene assay for early-stage breast cancer. Am J Manag Care 2011;17(5 Spec No):e194-e202.

28. Geffen DB, Abu-Ghanem S, Sion-Vardy N, et al. The impact of the 21-gene recurrence score assay on decision making about adjuvant chemotherapy in early-stage estrogen-receptor-positive breast cancer in an oncology practice with a unified treatment policy. Ann Oncol 2011;22:2381-2386.

29. Klang SH, Hammerman A, Liebermann N, Efrat N, Doberne J, Hornberger J. Economic implications of 21-gene breast cancer risk assay from the perspective of an Israeli-managed health-care organization. Value Health 2010;13:381-387.

30. Asad J, Jacobson AF, Estabrook A, et al. Does oncotype DX recurrence score affect the management of patients with early-stage breast cancer? Am J Surg 2008;196:527-529.

31. Oratz R, Paul D, Cohn AL, Sedlacek SM. Impact of a commercial reference laboratory test recurrence score on decision making in early-stage breast cancer. J Oncol Pract 2007;3:182-186.

32. Kelly CM, Krishnamurthy S, Bianchini G, et al. Utility of oncotype DX risk estimates in clinically intermediate risk hormone receptor-positive, HER2-normal, grade II, lymph node-negative breast cancers. Cancer 2010;116:5161-5167. 
33. Henry LR, Stojadinovic A, Swain SM, Prindiville S, Cordes R, Soballe PW. The influence of a gene expression profile on breast cancer decisions. J Surg Oncol 2009;99:319-323.

34. Tsoi DT, Inoue M, Kelly CM, Verma S, Pritchard KI. Cost-effectiveness analysis of recurrence score-guided treatment using a 21-gene assay in early breast cancer. Oncologist 2010;15:457-465.

35. Kondo M, Hoshi SL, Yamanaka T, Ishiguro H, Toi M. Economic evaluation of the 21-gene signature (Oncotype DX) in lymph node-negative/positive, hormone receptor-positive early-stage breast cancer based on Japanese validation study (JBCRG-TR03). Breast Cancer Res Treat 2011;127: 739-749.

36. Drummond M, Barbieri M, Cook J, et al. Transferability of economic evaluations across jurisdictions: ISPOR Good Research Practices Task Force report. Value Health 2009;12:409-418.
37. Partin JF, Mamounas EP. Impact of the 21-gene recurrence score assay compared with standard clinicopathologic guidelines in adjuvant therapy selection for node-negative, estrogen receptor-positive breast cancer. Ann Surg Oncol 2011;18:3399-3406.

38. Thompson D, Taylor DC, Montoya EL, Winer EP, Jones SE, Weinstein MC. Cost-effectiveness of switching to exemestane after 2 to 3 years of therapy with tamoxifen in postmenopausal women with early-stage breast cancer. Value Health 2007;10:367-376.

39. Gold MR, Siegel JE, Russell LB, Weinstein MC (eds). Cost-Effectiveness in Health and Medicine. Oxford University Press: New York, NY, 1996.

40. US Bureau of Labor Statistics Consumer Price Index-All Urban Consumers. http://data.bls.gov/cgi-bin/surveymost?cu. Accessed 26 January 2011. 